\title{
Probing molecular interactions on carbon nanotube surfaces using surface plasmon resonance sensors
}

Nurbek Kakenov, Osman Balci, Sinan Balci, and Coskun Kocabas

Citation: Appl. Phys. Lett. 101, 223114 (2012);

View online: https://doi.org/10.1063/1.4768927

View Table of Contents: http://aip.scitation.org/toc/apl/101/22

Published by the American Institute of Physics

\section{Articles you may be interested in}

Plasmon-polaritons on graphene-metal surface and their use in biosensors

Applied Physics Letters 100, 213110 (2012); 10.1063/1.4721453

Localization of surface plasmon polaritons in hexagonal arrays of Moiré cavities

Applied Physics Letters 98, 031101 (2011); 10.1063/1.3529469

Contact resistance between metal and carbon nanotube interconnects: Effect of work function and wettability Applied Physics Letters 95, 264103 (2009); 10.1063/1.3255016

Erratum: Probing molecular interactions on carbon nanotube surfaces using surface plasmon resonance sensors [Appl. Phys. Lett. 101, 223114 (2012)]

Applied Physics Letters 107, 189903 (2015); 10.1063/1.4935264

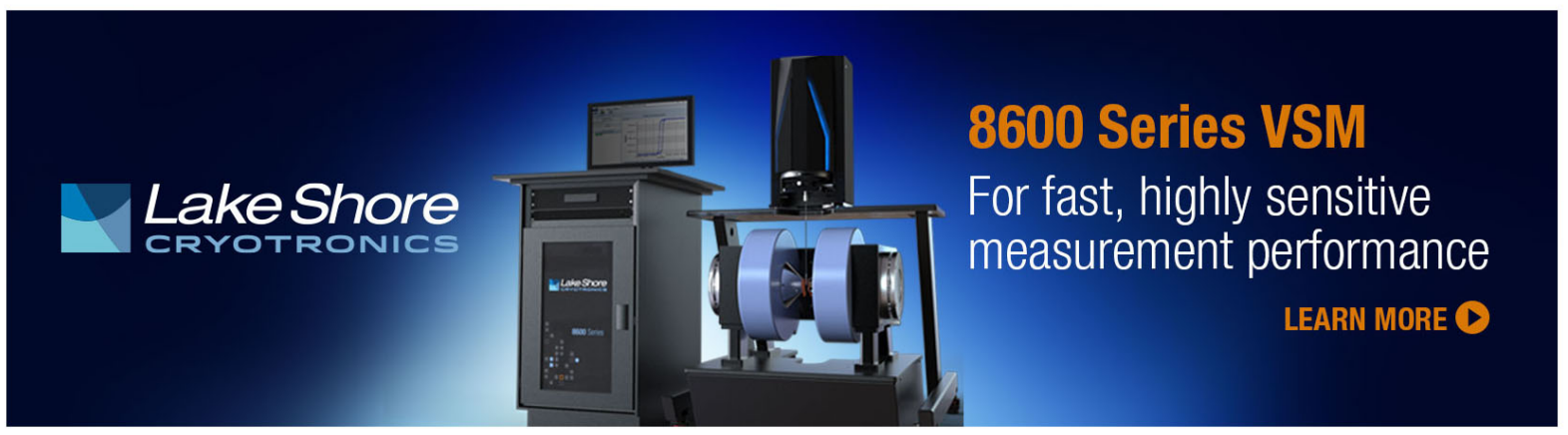




\title{
Probing molecular interactions on carbon nanotube surfaces using surface plasmon resonance sensors
}

\author{
Nurbek Kakenov, ${ }^{1}$ Osman Balci, ${ }^{1}$ Sinan Balci, ${ }^{2}$ and Coskun Kocabas ${ }^{1, a)}$ \\ ${ }^{1}$ Department of Physics, Bilkent University, 06800 Ankara, Turkey \\ ${ }^{2}$ Department of Mechanical Engineering, University of Turkish Aeronautical Association, 06790 Ankara, \\ Turkey
}

(Received 13 July 2012; accepted 9 November 2012; published online 30 November 2012)

\begin{abstract}
In this work, we present a method to probe molecular interactions on single-walled carbon nanotube (SWNT) surfaces using a surface plasmon sensor. SWNT networks were synthesized by chemical vapor deposition and transfer-printed on gold surfaces. We studied the excitation of surface plasmon-polaritons on nanotube coated gold surfaces with sub-monolayer, monolayer, and multilayer surface coverage. Integrating the fabricated sensor with a microfluidic device, we were able to obtain binding dynamics of a bovine serum albumin (BSA) protein on SWNT networks with various tube densities. The results reveal the kinetic parameters for nonspecific binding of BSA on SWNT coated surfaces having various tube densities. (C) 2012 American Institute of Physics. [http://dx.doi.org/10.1063/1.4768927]
\end{abstract}

The surface of low-dimensional carbon (carbon nanotubes and graphene) has unique electronic properties due to the delocalized p-orbitals. Very high carrier mobility together with nanoscale dimension makes carbon nanotubes and graphene promising candidates for high performance electronics applications. ${ }^{1}$ Besides unique electronic properties, the delocalized orbital has a strong tendency to adsorb aromatic molecules via p-electronic interactions. The strong non-covalent interactions between the graphitic surface and organic molecules provide a unique template for supramolecular chemistry ${ }^{2,3}$ and sensing applications. ${ }^{4,5}$ A comprehensive understanding of these forces at atomic and molecular level still remains a challenge. A great deal of computational $^{6,7}$ and experimental effort ${ }^{3,5,7,8}$ has been devoted to elucidate these interactions. New techniques with improved sensitivities are needed to probe the dynamics of organic molecules on the surface of low-dimensional carbon nanomaterials.

In the literature, adsorption of molecules on carbon surfaces has been studied extensively. The earlier works are based on bulk measurements using sorption isotherms ${ }^{9}$ and calorimetric studies. ${ }^{10}$ Recently, sensitive techniques have been demonstrated. Schedin et al. ${ }^{11}$ have demonstrated detection of a single molecule adsorbed on graphene surface by tracing changes in the Hall resistivity of graphene. Barone et al. $^{12}$ have demonstrated near-infrared optical sensors based on modulation of emission of single-walled carbon nanotube (SWNT) in response to adsorbed biomolecules. Electrical response of SWNTs in a transistor geometry was also used for detecting adsorbed molecules. ${ }^{4,13}$ Carbon nanotube based capacitive sensors ${ }^{14}$ electrochemical sensors, ${ }^{15}$ and flow sensors ${ }^{16}$ are good examples for detecting adsorbed molecules on nanotube surface.

In this work, we integrated surface plasmon resonance (SPR) sensors with networks of single-walled carbon nanotubes to study interactions between SWNT and organic mol-

\footnotetext{
${ }^{\text {a) }}$ Author to whom correspondence should be addressed. Electronic mail: ckocabas@fen.bilkent.edu.tr. Telephone: +90(312)2901965.
}

ecules. SPR sensors provide surface specific detection schemes with superior sensitivity. The evanescent field of surface plasmons (SPs) decays exponentially in strength with distance away from the surface with a decay length of a few hundreds of nanometers. Surface specific detection together with the high sensitivity allows the widespread acceptance of SPR sensors. Here, we implement SPR sensors to probe dynamics of a medium size protein on SWNT surfaces. SWNT networks with various tube densities were grown by chemical vapor deposition and then transfer-printed on gold surfaces. To understand the effects of SWNT network on plasmonic properties of the surface, we first measured the resonance characteristics of surface plasmon-polaritons (SPPs) on SWNT functionalized gold surface. As a demonstration of SWNT modified SPR sensor we studied binding dynamics of bovine serum albumin (BSA) protein on SWNT network with various tube densities.

The SWNT networks were grown by catalytic chemical vapor deposition technique using iron nanoparticles as seeds for the nanotube growth. The SWNT growth process starts with coating a silicon wafer with a $500 \mathrm{~nm}$ thick photoresist (Shipley 1805) doped with $\mathrm{Fe}_{2} \mathrm{Cl}_{3}$ having concentration of 1-3 mM. Annealing the photoresist in air results in dispersed iron oxide nanoparticles on the $\mathrm{SiO}_{2}$ surface. Iron oxide particles are reduced by hydrogen $(300 \mathrm{sccm})$ as the temperature of the samples reaches to $920^{\circ} \mathrm{C}$. After reducing the iron oxide nanoparticles, we started the SWNT growth by flowing ethanol vapor into the quartz chamber using a carrier flow of $50 \mathrm{sccm} \mathrm{Ar}$ and $50 \mathrm{sccm} \mathrm{H}_{2}$ at $920^{\circ} \mathrm{C}$. After $15 \mathrm{~min}$, we terminated the growth and cooled the samples back to the room temperature under a flow of Ar. The density of SWNT networks was controlled by changing the concentration of $\mathrm{Fe}_{2} \mathrm{Cl}_{3}$ in the photoresist layer. Following the growth process, we transferred SWNTs on metal substrates. Growing SWNT directly on $50 \mathrm{~nm}$ gold surface is not possible because of dewetting of gold layer on $\mathrm{SiO}_{2}$ surface. ${ }^{17}$ In order to solve this problem, we developed a transfer printing process for transferring $\mathrm{SWNTs}$ from $\mathrm{SiO}_{2}$ coated $\mathrm{Si}$ substrates to metal substrates. Fig. 1 shows the steps of the 


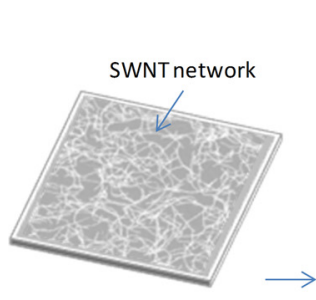

CVD of SWNT network

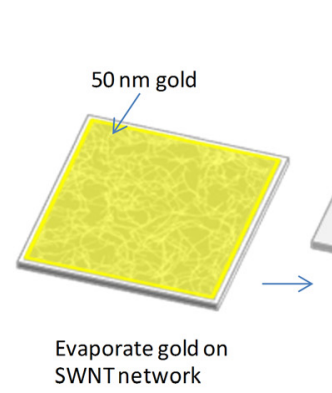
SWNT network

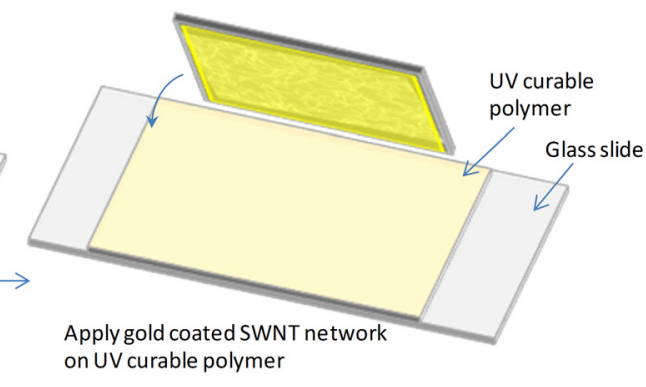

Expose UV light

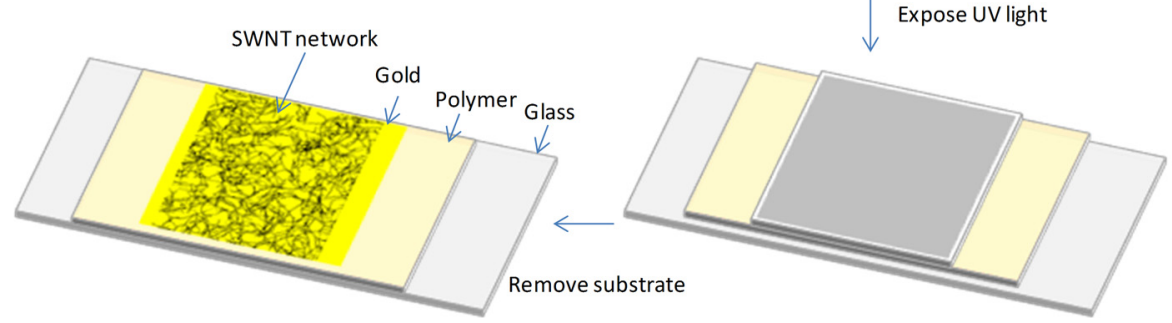

FIG. 1. Transfer printing process of SWNT network grown on $\mathrm{SiO}_{2}$ coated Si substrates. transfer printing process of SWNT networks to the gold surface. We first evaporated $50 \mathrm{~nm}$ thick gold film on the SWNT network. Thick gold film of $50 \mathrm{~nm}$ satisfies the critical coupling condition, which provides the highest sensitivity for the sensor. The role of the gold layer is twofold; first, it provides mechanical support for SWNT during the transfer printing process with low adhesion on $\mathrm{SiO}_{2}$ surface, second, it supports surface plasmon-polaritons. We applied the gold coated surface on a glass slide coated with UV curable polymer (Epo-Tek, OG146). A 5-min exposure of UV light cures the polymer layer and sticks the gold-SWNT layer to the cured polymer surface. The SWNT networks on gold surface are achieved by mechanically removing the Si substrate with a razorblade.

Fig. 2 shows the scanning electron micrographs of SWNT networks with various tube densities on $\mathrm{SiO}_{2}$ substrates. The tube density was controlled by changing the catalyst concentration. The surface coverage of the SWNT networks ranges from submonolayer ( $\sim 5 \%$ coverage) to multilayer. We named the three representative tube densities as D1 (submonolayer), D2 (monolayer), and D3 (multilayer). The tube densities for D1, D2, and D3 are $\sim 1 \mathrm{SWNT} / \mu \mathrm{m}^{2}$, $\sim 10 \mathrm{SWNT} / \mu \mathrm{m}^{2}$, and $\sim 50 \mathrm{SWNT} / \mu \mathrm{m}^{2}$, respectively. The carbon nanotubes are primarily single-walled with tube diameters ranging from $0.7 \mathrm{~nm}$ to $4 \mathrm{~nm}$.

Before studying the binding kinetics of the organic molecules, we first characterized the plasmonic properties of the gold surface coated with varying SWNT network density. Fig. 3 shows the experimental setup used to excite SPPs. Due to the confined nature of SPPs, their momentum is larger than the momentum of light in free space. The momentum mismatch can be compensated using a prism with high index of refraction. This arrangement is known as Kretschmann configuration. A supercontinium laser attached with an acousto-optical modulator (Koheras, SuperK versa) was used as a tunable light source with a spectral width of $\sim 1 \mathrm{~nm}$. Surface plasmon-polaritons are excited by TM polarized light at the resonance angle where the phase matching condition is satisfied as

$$
\sin \left(\theta_{c}\right)=\frac{k_{s p p}}{n_{p} k_{0}}=\frac{1}{n_{p}} \sqrt{\frac{\varepsilon_{m} \varepsilon_{d}}{\varepsilon_{m}+\varepsilon_{d}}},
$$

where $k_{s p p}$ is the momentum of surface plasmon-polaritons, $n_{p}$ is the refractive index of the prism, $k_{0}$ is the momentum of light in free space, $\varepsilon_{m}$ and $\varepsilon_{d}$ are the dielectric constant of the metal and the medium, respectively. The refractive index of the prism is $\sim 1.52$. The $50 \mathrm{~nm}$ thick gold layer used during the transfer printing process supports the SPPs. In order to obtain the dispersion curves, we measured the polarization dependent reflectivity of the surface as a function of wavelength (from $400 \mathrm{~nm}$ to $1000 \mathrm{~nm}$ ) and incidence angle (from $40^{\circ}$ to $48^{\circ}$ ) with a precision of less than $1 \mathrm{~nm}$ and $0.01^{\circ}$, respectively.
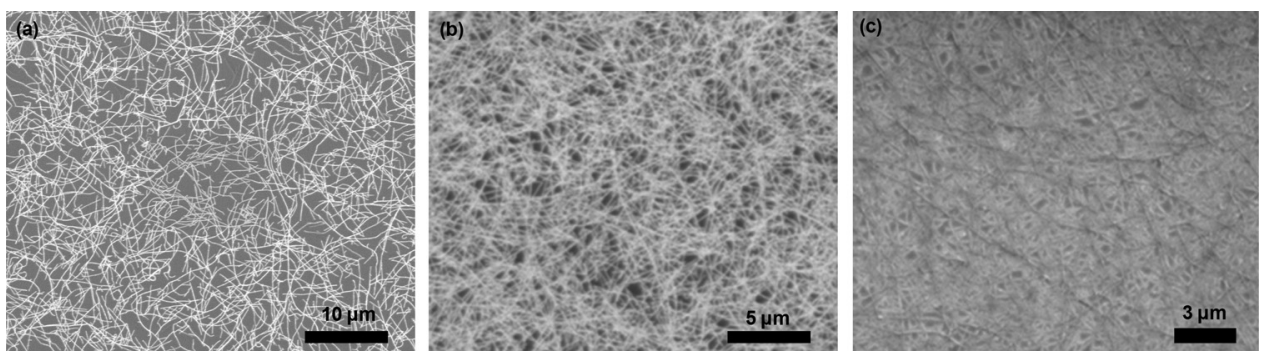

FIG. 2. Electron micrographs of SWNT networks with various tube densities grown by chemical vapor deposition on $\mathrm{SiO}_{2}$ substrates. Tube density was controlled by changing the concentration of the catalyst. The varying amount of nanotube network is named as sub-monolayer $\left(\mathrm{D} 1=1 \mathrm{SWNT} / \mu \mathrm{m}^{2}\right)$, monolayer $\left(\mathrm{D} 2=10 \mathrm{SWNT} / \mu \mathrm{m}^{2}\right)$, and multilayer $\left(\mathrm{D} 3=50 \mathrm{SWNT} / \mu \mathrm{m}^{2}\right)$, respectively. The tube diameter ranges from $0.7 \mathrm{~nm}$ to $4 \mathrm{~nm}$. 


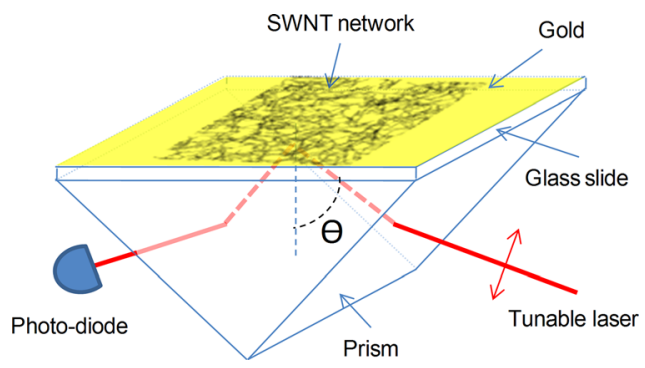

FIG. 3. Experimental setup (Kretschmann configuration) used to excite SPP on metal surface coated with SWNT network. The thickness of the gold layer is $50 \mathrm{~nm}$. The prism is mounted on a double rotary state. The reflected beam is detected by a photodiode. The incidence angle and the wavelength of the laser are controlled with a precision of less than $0.01^{\circ}$ and $1 \mathrm{~nm}$, respectively.

Fig. 4 shows the reflectivity maps from the SWNT coated gold surfaces as a function of excitation wavelength and incidence angle. The wavelength scans the energy of the exited SPPs and the angle defines the momentum. The maps were obtained from the TM polarized reflection spectra. When the resonance condition (Eq. (1)) is satisfied, the reflectivity goes to a minimum value. The resonance wavelength is obtained from the minimum value. We observed that as the surface coverage increases, the resonance wavelength red-shifts and the spectral width of the surface plasmon resonance gets broader, which is likely because of additional optical losses (scattering losses) associated with the high density of SWNT networks.

The characteristic surface plasmon absorption dip can be seen not only by scanning the wavelength of the excitation, but also it can be obtained by scanning the incidence angle with monochromatic excitation. Fig. 5 summarizes the variation in the reflection spectra with increasing tube density. Fig. 5(a) shows the reflection spectra of the surface recorded at an incidence angle of $44^{\circ}$. There are two resonances; the one that is at around $470 \mathrm{~nm}$ is due to the bulk plasmon absorption, the second resonance at around $605 \mathrm{~nm}$ is due to the surface plasmon-polaritons. The bulk plasmon absorption is independent of the surface coverage. Angular dependence of the reflectivity from the surfaces is shown in Fig. 5(b). The resonance angle increases with increasing the nanotube density. Figs. 5(c) and 5(d) show the variation of resonance wavelength and resonance angle as a function of tube density, respectively.

The gold surface functionalized with SWNT network provides us a unique platform to probe binding interaction between SWNT surface and organic molecules. As a proof of principle, we studied nonspecific binding of a BSA protein on SWNT surface. In order to probe binding interactions of proteins on SWNT network, we integrated the SPR sensor with a microfluidic device. Fig. 6(a) shows the fabrication steps of the microfluidic device. The device consists of an elastomeric flow chamber sealed on the SWNT functionalize gold surface. For an efficient coupling of SPP, the thickness of the gold layer is chosen to be $50 \mathrm{~nm}$. A TM polarized diode laser $(\lambda=635 \mathrm{~nm})$ and a prism with a high index of refraction $\left(\mathrm{BK} 7, \mathrm{n}_{\mathrm{p}}=1.78\right)$ are used to excite SPPs. The reflectivity as a function of incidence angle is given in Fig. 6(b). The SPR angle is at $57^{\circ}$ for $635 \mathrm{~nm}$ excitation wavelength. After setting the incidence angle to the resonance angle, we first run deionized (DI) water through the flow chamber for $5 \mathrm{~min}$. When the SPR signal was stabilized, we started the flow of $100 \mathrm{nM}$ BSA solution and recorded the SPR signal for $30 \mathrm{~min}$. We repeated the same procedure for a bare gold surface and gold surfaces coated with three different SWNT networks shown in Fig. 3. The time traces of SPR signal for binding interaction of BSA on the various surfaces are shown in Fig. 6(c). After the SPR signal saturated, we washed the surface with DI water. We did not observe any change in the SPR signal, which indicates that BSA is kinetically stable on SWNT surface.

To understand the nonspecific binding of BSA on SWNT network, we used a first-order pseudo-kinetic equation. Since we employed a flow cell, the number of molecules in the medium is much higher than the available binding sites on the surface. The pseudo-kinetic equation can be written as

$$
\frac{d A}{d t}=k_{a} C(\beta-A)-k_{d} A,
$$

where $A$ is the concentration of molecules binding to the surface, $k_{a}$ is the association rate constant, $\beta$ is the concentration of free binding sites, and $k_{d}$ is the dissociation rate constant. Since we did not observe any disassociation from the surface, the value of $k_{d}$ is zero. With these conditions, the solution of the kinetic equation is

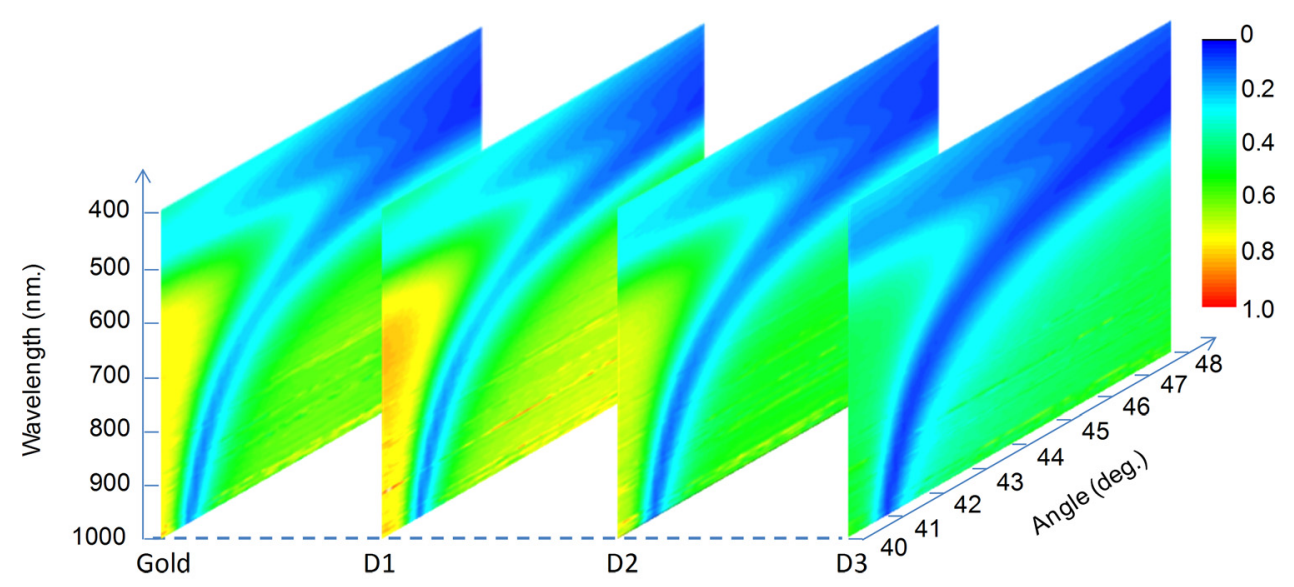

FIG. 4. The reflectivity maps (angular dispersion curves) from the SWNT coated gold surface as a function of incidence angle and the excitation wavelength. The light source is TM polarized. As the density of SWNTs network increases, the effective index of the surface plasmon-polaritons increases resulting in a red shift in the plasmon resonance wavelength. The color map shows the scale for the reflectivity. 

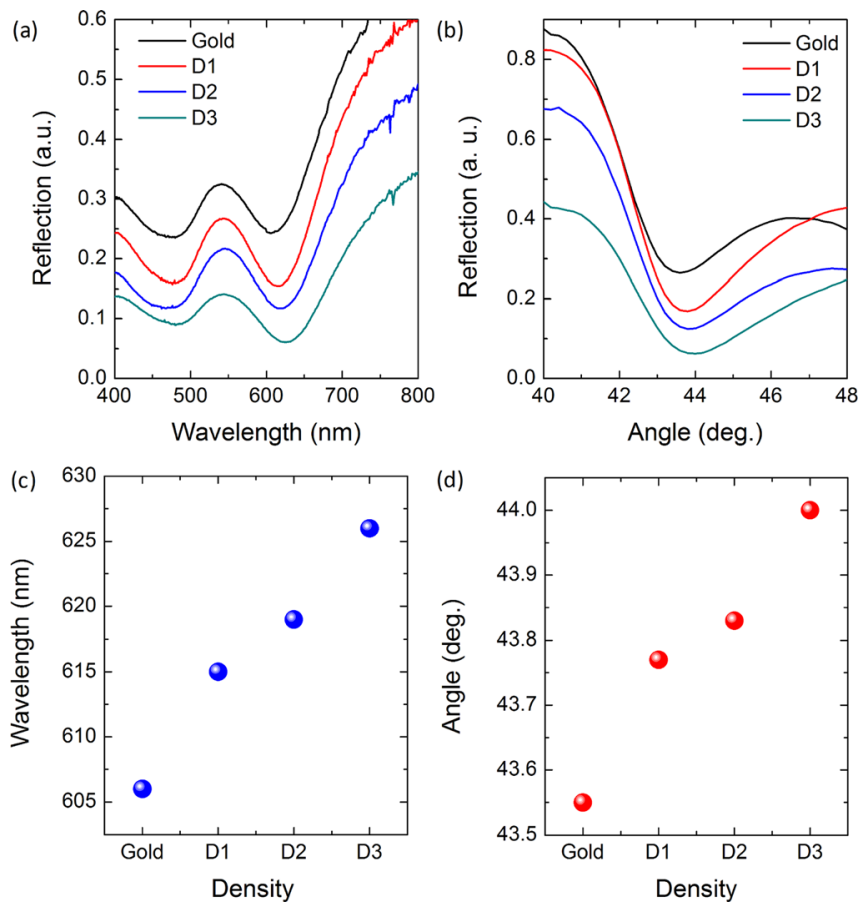

FIG. 5. (a) Reflection spectra from the gold surface with various tube densities. The incidence angle is $44^{\circ}$. There are two resonances in the reflectivity spectra; bulk plasmon resonance of around $450 \mathrm{~nm}$ and surface plasmon resonance of around $600 \mathrm{~nm}$. (b) Reflectivity of the surface as a function of incidence angle for various nanotube coverages. (c) and (d) Dependence of surface plasmon resonance wavelength and angle on the surface coverage of SWNT networks, respectively.

$$
C(t)=\beta\left(1-e^{-k_{a} A t}\right)
$$

In the linear regime of the reflection spectrum, the SPR signal is proportional to the concentration of molecules binding to the surface. We obtained $\beta$ and $k_{a}$ values from the least squares fitting of the time trace of SPR signal for various SWNT networks. Fig. 6(d) shows the extracted values of $\beta$ which quantifies the concentration of free binding sites. The concentration of free binding sites increases with the tube density while the association constant stays constant with a value of $0.56 \times 10^{5} \mathrm{M}^{-1} \mathrm{~s}^{-1}$. The mechanism behind the adsorption of organic molecules on carbon surfaces from an aqueous solution is not fully understood. The interplay between hydrophobic interactions, electrostatic interactions, and hydrogen bonds determines the binding kinetics. Furthermore, the mechanism is highly dependent on the heterogeneity of the surface. Since, the surface of SWNTs is hydrophobic, hydrophobic interactions play the main role of nonspecific binding on carbon based surfaces. The hydrophobicity of metal surface coated by the SWNT networks changes with the tube density. BSA has several hydrophobic binding sites. As the surface of the sensor covered by SWNTs, the available binding sites increase resulting in an increase in the effective association constant. The SWNT networks were synthesized by catalytic chemical vapor deposition method using iron oxide nanoparticles. This method generated SWNT with a diameter distribution between $0.7 \mathrm{~nm}$ and $3 \mathrm{~nm}$. Our previous analysis showed that the synthesized nanotubes are single-walled nanotubes; however, small fraction of them forms bundles and double walled nanotube. The presented method provides an ensemble aver-
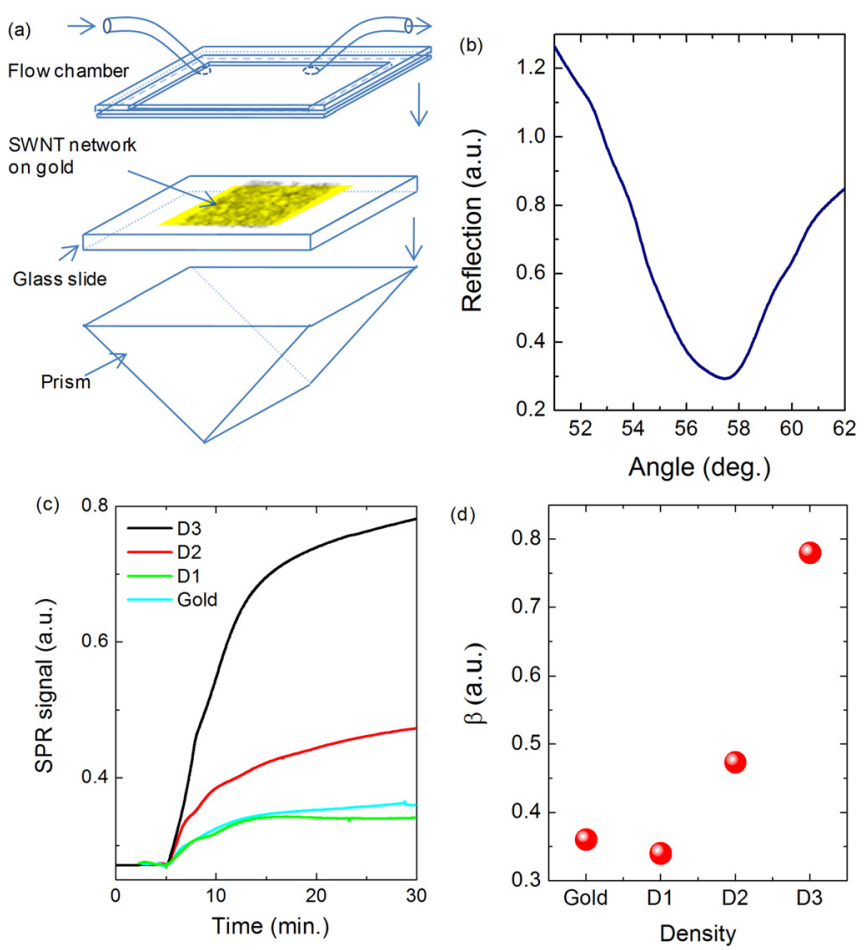

FIG. 6. (a) Schematic representation of the microfluidic device integrated with SPR sensor functionalized with SWNT network. The flow chamber is sealed on SWNT coated gold surface. The glass slide is attached to the prism using an index matching fluid. (b) Reflectivity of the gold surface as a function of incidence angle at $635 \mathrm{~nm}$. The resonance angle is $57^{\circ}$. (c) Time trace of SPR signal for binding interaction of BSA on gold surface coated with various SWNT network. The concentration of BSA is $100 \mathrm{nM}$. (d) The extracted parameter $(\beta)$ quantifies the available binding sites on the surface of SPR sensor. The $\beta$-parameter for gold and submonolayer SWNT networks is around 0.35 . As the density of SWNT increases the $\beta$-parameter changes from 0.35 to 0.8 while the association constant stays constant with a value of $0.56 \times 10^{5} \mathrm{M}^{-1} \mathrm{~s}^{-1}$.

aged binding dynamics on large number of SWNTs with different chirality and diameters. Similar experiments can be done on single tube level to elucidate the effects of chirality and diameter of SWNTs on binding dynamics.

As a conclusion, we present a SWNT modified SPR sensor to probe binding dynamics of organic molecules on SWNT surfaces. SWNT networks with various tube densities were grown by chemical vapor deposition and then transferprinted on gold surfaces. We characterized the plasmonic properties of gold surfaces having varying amount of SWNT densities. As a demonstration of SWNT modified SPR sensor we studied binding dynamics of bovine serum albumin protein on SWNT networks with various tube densities. We observed that the concentration of free binding sites increases with the tube density while the association constant stays constant with the value of $0.56 \times 10^{5} \mathrm{M}^{-1} \mathrm{~s}^{-1}$. We anticipate that the extreme sensitivity of SPR sensors together with special signal interrogation techniques could allow us to detect adsorption of small molecules even on a single SWNT.

This work was supported by the Scientific and Technological Research Council of Turkey (TUBITAK) (Grant Nos. 110T304 and 109T209), Marie Curie International Reintegration Grant (IRG) (Grant No. 256458), Turkish Academy of Science (TUBA-Gebip). 
${ }^{1}$ C. Wang, A. Badmaev, A. Jooyaie, M. Q. Bao, K. L. Wang, K. Galatsis, and C. W. Zhou, ACS Nano 5(5), 4169 (2011); E. Pince and C. Kocabas, Appl. Phys. Lett. 97(17), 173106 (2010); Y. M. Lin, A. Valdes-Garcia, S. J. Han, D. B. Farmer, I. Meric, Y. N. Sun, Y. Q. Wu, C. Dimitrakopoulos, A. Grill, P. Avouris, and K. A. Jenkins, Science 332(6035), 1294 (2011); C. Kocabas, H. S. Kim, T. Banks, J. A. Rogers, A. A. Pesetski, J. E. Baumgardner, S. V. Krishnaswamy, and H. Zhang, Proc. Natl. Acad. Sci. U.S.A. 105(5), 1405 (2008); S. J. Kang, C. Kocabas, T. Ozel, M. Shim, N. Pimparkar, M. A. Alam, S. V. Rotkin, and J. A. Rogers, Nat. Nanotechnol. 2(4), 230 (2007); C. Rutherglen, D. Jain, and P. Burke, ibid. 4(12), 811 (2009).

${ }^{2}$ J. P. Hill, W. Jin, A. Kosaka, T. Fukushima, H. Ichihara, T. Shimomura, K. Ito, T. Hashizume, N. Ishii, and T. Aida, Science 304(5676), 1481 (2004); Y. Zhao and D. G. Truhlar, J. Am. Chem. Soc. 129(27), 8440 (2007).

${ }^{3}$ S. Grimme, J. Antony, T. Schwabe, and C. Muck-Lichtenfeld, Org. Biomol. Chem. 5(5), 741 (2007).

${ }^{4}$ J. Kong, N. R. Franklin, C. W. Zhou, M. G. Chapline, S. Peng, K. J. Cho, and H. J. Dai, Science 287(5453), 622 (2000); B. L. Allen, P. D. Kichambare, and A. Star, Adv. Mater. 19(11), 1439 (2007).

${ }^{5}$ O. Salihoglu, S. Balci, and C. Kocabas, Appl. Phys. Lett. 100(21), 213110 (2012).

${ }^{6}$ O. Gulseren, T. Yildirim, and S. Ciraci, Phys. Rev. Lett. 87(11), 116802 (2001); E. Durgun, S. Dag, V. M. K. Bagci, O. Gulseren, T. Yildirim, and S. Ciraci, Phys. Rev. B 67(20), 201401 (2003); P. A. Denis and F. Iribarne, J. Mol. Struct.: THEOCHEM 957(1-3), 114 (2010).

${ }^{7}$ E. Durgun, S. Dag, S. Ciraci, and O. Gulseren, J. Phys. Chem. B 108(2), 575 (2004).
${ }^{8}$ S. B. Fagan, A. G. Souza, J. O. G. Lima, J. Mendes, O. P. Ferreira, I. O. Mazali, O. L. Alves, and M. S. Dresselhaus, Nano Lett. 4(7), 1285 (2004); L. L. Wang, D. Q. Zhu, L. Duan, and W. Chen, Carbon 48(13), 3906 (2010); B. Pan and B. S. Xing, Environ. Sci. Technol. 42(24), 9005 (2008); E. S. Alldredge, S. C. Badescu, N. Bajwa, F. K. Perkins, E. S. Snow, T. L. Reinecke, J. L. Passmore, and Y. L. Chang, Phys. Rev. B 78(16), 161403 (2008).

${ }^{9}$ K. Yang, X. Wang, L. Zhu, and B. Xing, Environ. Sci. Technol. 40(18), 5804 (2006); S. Debnath, Q. H. Cheng, T. G. Hedderman, and H. J. Byrne, J. Phys. Chem. C 114(18), 8167 (2010); A. Patrykiejew, M. Jaroniec, and A. W. Marczewski, Thin Solid Films 76(3), 247 (1981).

${ }^{10}$ E. Castillejos, B. Bachiller-Baeza, I. Rodriguez-Ramos, and A. GuerreroRuiz, Carbon 50(8), 2731 (2012).

${ }^{11}$ F. Schedin, A. K. Geim, S. V. Morozov, E. W. Hill, P. Blake, M. I. Katsnelson, and K. S. Novoselov, Nature Mater. 6(9), 652 (2007).

${ }^{12}$ P. W. Barone, S. Baik, D. A. Heller, and M. S. Strano, Nature Mater. 4(1), 86 (2005).

${ }^{13}$ P. G. Collins, K. Bradley, M. Ishigami, and A. Zettl, Science 287(5459), 1801 (2000).

${ }^{14}$ E. S. Snow, F. K. Perkins, E. J. Houser, S. C. Badescu, and T. L. Reinecke, Science 307(5717), 1942 (2005).

${ }^{15}$ Q. Zhao, Z. H. Gan, and Q. K. Zhuang, Electroanalysis 14(23), 1609 (2002).

${ }^{16}$ S. Ghosh, A. K. Sood, and N. Kumar, Science 299(5609), 1042 (2003).

${ }^{17}$ T. Oznuluer, E. Pince, E. O. Polat, O. Balci, O. Salihoglu, and C. Kocabas, Appl. Phys. Lett. 98(18), 183101 (2011). 